\title{
Morphologie des spermatozoïdes
}

\author{
Mieusset R.
}

Centre de Stérilité Masculine, Hôpital de la Grave, 31052 Toulouse Cedex

\section{RESUME}

La morphologie des spermatozoïdes est l'un des paramètres qualitatifs de la spermatogenèse. En raison des différentes techniques utilisées, de la définition de la normalité et des différents types d'anomalies, la morphologie reste trop souvent une caractéristique mal utilisée en pratique courante. Les anomalies morphologiques peuvent être d'origine soit testiculaire, soit, mais cela reste à prouver, d'origine post-testiculaire. L'étiologie de ces anomalies est d'ordre génétique, et dans ce cas tous les gamètes sont atteints, ou constituée par une pathologie endogène dont l'exemple le plus souvent évoqué, bien que non formellement confirmé, est le varicocèle. Mais il existe aussi des facteurs exogènes qui induisent des anomalies morphologiques, qu'ils soient de nature chimique comme certains médicaments ou insecticides, ou physique comme par exemple la chaleur. L'analyse de la morphologie des spermatozoïdes apporte des résultats qui ont une utilité à la fois comme indicateur de santé du testicule (lors d'exposition à des agents chimiques ou physiques d'ordre professionnel) et comme indicateur de la fertilité potentielle, puisque la morphologie est corrélée à la mobilité des gamètes, et impliquée dans la fécondation à travers la réaction acrosomique.
Mots Clés : Spermatozoïdes - Morphologie Fécondité - Toxicologie.

\section{INTRODUCTION}

Les paramètres qui caractérisent les spermatozoïdes se répartissent en deux groupes, quantitatif avec la numération, qualitatif avec la vitalité, la mobilité et la morphologie. Si les deux premières caractéristiques de la qualité, vitalité et mobilité, renvoient à des valeurs chargées "positivement" dans notre société (dynamisme, entreprise, mobilité sociale ...) et dans la représentation individuelle (ce que je produis est plein de vie et de mouvement, mon enfant sera plein de santé), la morphologie des spermatozoïdes est principalement abordée sous un angle négatif, puisqu'il s'agit essentiellement d'une description et d'une énumération des formes anormales, c'est-à-dire la mise en évidence d'anomalies, de monstruosités, qui renvoient d'une part à la norme sociale, avec tout ce qu'elle peut avoir de subjectif, et donc de fluctuant, d'imprécis, voire de parti pris, et d'autre part individuellement à l'angoissante question de la survenue d'un enfant tout aussi mal formé que les spermatozoïdes.

Nous aborderons successivement, après avoir rappelé ce qu'est l'analyse morphologique des spermatozoïdes, l'origine puis les causes potentielles des anomalies morphologiques des gamètes mâles. Nous 
terminerons en présentant deux utilisations possibles de l'analyse morphologique, à savoir comme indicateur de fécondité in vivo et comme indicateur de "santé".

\section{QU'EST-CE QUE L'ANALYSE MORPHOLOGIQUE DES SPERMATOZOIDES ?}

C'est l'étude descriptive de la forme des spermatozoïdes à partir d'un échantillon représentatif d'un éjaculat, à partir d'un étalement sur lame puis coloration des spermatozoïdes fixés. Le résultat de cette analyse est la distribution des spermatozoïdes en deux groupes, les normaux et les anormaux, ces derniers faisant par ailleurs l'objet d'une classification selon les types d'anomalies.

Cette analyse morphologique soulève de nombreuses questions méthodologiques comprenant la variabilité du sperme et les facteurs techniques de variation, la variabilité des résultats d'une équipe à l'autre (voir D. Clavert et A. Bourguignat), la définition des critères de normalité selon la technique utilisée (voir D. Le Lanou). Toutes ces questions sont loin d'être résolues actuellement. Et quelles que soient les incertitudes et les imprécisions des différentes techniques d'analyse et d'objets étudiés, elles font toutes apparaître une liste d'anomalies qui caractérisent les spermatozoïdes anormaux.

Rappelons enfin une notion encore trop mal connue : la morphologie des spermatozoïdes n'est pas un paramètre constant dans l'éjaculat d'un homme donné : la démonstration en a été faite sur 5 éjaculats successifs chez 10 hommes féconds [45] et sur 5 éjaculats mensuels chez 45 hommes fertiles [44]. Aucune analyse n'a été rapportée chez les hommes inféconds quant à la stabilité du pourcentage de formes normales, ni des types d'anomalies.

\section{ORIGINE DES ANOMALIES \\ MORPHOLOGIQUES DES SPERMATOZOIDES}

Ces anomalies peuvent être classées en deux grands groupes selon leur lieu de survenue testiculaire ou post-testiculaire.

\section{Les anomalies testiculaires}

Ce sont des malformations qui surviennent lors de la spermatogenèse

a) le plus souvent lors de la spermiogénèse, avec au niveau :

- de la tête : les anomalies de forme du noyau (allongé, aminci) ou l'absence d'acrosome [14];

- de la pièce intermédiaire : les angulations du flagelle, qui sont une implantation anormale de la pièce connective sur la tête ou une anomalie de relation topographique entre capitulum et colonnes segmentées [49], mais aussi l'absence apparente ou la finesse de la pièce intermédiaire qui donne l'impression que la pièce principale s'implante directement dans la tête [49]. Cette dernière anomalie traduit une absence de mitochondries au niveau de la PI, anomalies survenant aux derniers stades de la spermiogénèse (spermatides âgées, [42]).

- de la pièce principale avec les flagelles courts ou irréguliers (15).

b) mais aussi lors des phases antérieures à la spermiogénèse comme les spermatozoïdes à double tête, traduisant une anomalie de la mitose des spermatocytes II, ou les spermatozoïdes macrocéphales [13].

\section{Les anomalies post-testiculaires}

Elles surviendraient lors du transit et/ou du stockage épididymaire; ce sont alors des déformations, elles atteignent plutôt le flagelle. Ont été citées dans ce groupe, les flagelles isolés, les têtes en lyse, et les flagelles enroulés. Toutefois, certains auteurs 
avancent l'absence de toute altération morphologique lors du transit et/ou stockage épididymaire [18].

\section{CAUSES POTENTIELLES DE CES ANOMALIES}

On peut distinguer 3 types de causes différentes à ces anomalies :

\section{Anomalies génétiques}

Une participation génétique a été rapportée en ce qui concerne des anomalies morphologiques spécifiques : il s'agit des spermatozoïdes avec absence d'acrosome [17] et des pinhead spermatozoïdes ou spermatozoïdes sans tête [2]. On doit noter toutefois que pour être d'origine génétique, cette anomalie doit toucher l'ensemble des spermatozoïdes.

Les travaux de Martin et Rademaker [32] ont montré qu'il n'y avait pas de relation entre la proportion de spermatozoïdes morphologiquement anormaux et la proportion d'anomalies chromosomiques, que ce soit la fréquence totale des anomalies ou les anomalies chromosomiques numériques et structurales. A noter cependant que cette étude n'a été réalisée que chez des hommes féconds.

Bien qu'il ne semble pas exister de relation entre anomalies géniques et morphologiques [4], une étude récente [19] rapporte une élévation significative de plusieurs anomalies morphologiques des spermatozoïdes chez 25 pères d'un enfant avec hypospadias isolé par comparaison avec 50 pères appariés d'enfant sans hypospadias, l'hypospadias isolé étant attribué à une prédisposition génétique à expression favorisée par des facteurs environnementaux.

\section{Pathologies endogènes}

Un pourcentage élevé de spermatozoïdes de forme anormale a été évoqué dans le cas de varicocèle par certains auteurs [31], mais non retrouvé par d'autres [22]. Toutefois cette discordance entre les résultats des différentes études n'exclut en rien une altération de la forme des spermatozoïdes dans ces affections andrologiques. En effet, comme le pourcentage d'anomalies est très souvent associé à une infertilité, la référence pourrait se situer dans la comparaison de deux populations d'hommes féconds (dans lesquelles les pourcentages d'anomalies, quels qu'ils soient, ne sont pas source d'infécondité) avec et sans varicocèle.

\section{Pathologies exogènes}

Il s'agit des expositions à des facteurs exogènes ayant un effet délétère sur la forme des spermatozoïdes.

\section{a) Agents chimiques}

\section{- Médicaments}

Le plus connu d'entre eux est la sulfasalazine, utilisée dans le traitement de la $\mathrm{RCH}$, qui induirait entre autre des anomalies morphologiques des spermatozoïdes à type de macrocéphalie associée à une nucléomalacie $[25,46]$. L'affection en elle-même pourrait être impliquée dans ces anomalies par certains auteurs [24], mais la substitution par la mesalazine montre une récupération de la morphologie [40].

- Toxiques chimiques et industriels

- Insecticides : le carbamate (1-Naphtyl methyl) appelé aussi carbanyl ou Sevin induit (48) chez les employés des usines de fabrication une augmentation du pourcentage de formes anormales $(n=30$; $52 \%)$ comparé à un groupe témoin $(\mathrm{n}=34$; $42 \%$ ), cette augmentation persistant après l'arrêt de l'exposition $(\mathrm{n}=79 ; 50 \%)$. Le dibromoethane $[39,43]$ induit des modifications plus spécifiques, le pourcentage de formes anormales ne différant pas, mais les pourcentages de têtes amincies, de têtes absentes et d'anomalies flagellaires étant significativement augmentés chez les exposés ( $n=46)$ par rapport au non-exposés $(n=43)$. 
- Les soudeurs (aciers doux ou inoxydables) sont exposés aux fumées toxiques liées à la soudure. Ils ont un pourcentage de formes anormales augmenté par rapport aux témoins en ce qui concerne les aciers doux avec un pourcentage de formes anormales qui augmente significativement avec la durée d'exposition [5].

- Fibres artificielles : l'exposition au carbone disulphide induit des troubles sévères (polynévrites) ainsi qu'une tératozoospermie chez 31 hommes exposés comparés à 31 témoins [31]. Ceci ne fut pas confirmé par d'autres auteurs, mais le taux de participation à l'étude fut de $<50 \%$ chez les exposés [34].

- "Agent orange": les soldats américains ayant servi au Vietnam et exposés à des armes chimiques du type "agent orange" présentent des anomalies morphologiques caractéristiques, avec une réduction significative du pourcentage de spermatozoïdes à tête normale [10].

\section{- Agents physiques}

Une élévation induite de la température corporelle [38], scrotale [41] ou testiculaire [35] se traduit par une altération de la spermatogenèse incluant une augmentation du pourcentage de spermatozoïdes morphologiquement anormaux. Une élévation de $2^{\circ} \mathrm{C}$ de la température testiculaire induite 15 heures/jour se traduit en outre par une augmentation des anomalies de la tête (têtes allongées, amincies et à un moindre degré, irrégulières) et de la pièce intermédiaire, plus particulièrement les angulations du flagelle [35]. A noter que l'arrêt de l'exposition est suivi d'une récupération du pourcentage de formes normales, mettant ainsi en évidence qu'un facteur exogène peut ne pas avoir un effet irréversible sur la morphologie des spermatozoïdes. Enfin, certaines études épidémiologiques ont rapporté une augmentation du pourcentage de spermatozoïdes anormaux en cas d'exposition à de fortes températures en milieu professionnel $[6,16]$.

\section{UTILISATION DES RESULTATS DE \\ L'ANALYSE MORPHOLOGIQUE DES SPERMATOZOIDES}

Deux types d'utilisation sont possibles. La morphologie des spermatozoïdes peut être considérée comme un indicateur soit de la fertilité potentielle, soit de la qualité de la spermatogenèse mais indépendamment de la fécondité, c'est-à-dire comme un indicateur de santé.

\section{Fertilité potentielle}

Pour obtenir un embryon, les spermatozoïdes passent successivement par les étapes suivantes: traversée du mucus cervical; parcours dans utérus et trompe; passage des enveloppes d'ovule; réaction acrosomique et fusion avec ovocyte ; fécondation; développement d'un embryon.

Dans le cas de fécondation in vivo, les spermatozoïdes ont à franchir toutes ces étapes, la première étant contournée dans le cas d'insémination intra-utérine, les 3 ou les 2 dernières étant seules impliquées en cas de fécondation in vitro ou de micromanipulation des gamètes.

Pour réaliser les trois premières étapes, les spermatozoïdes doivent être mobiles. D'où une première question : quelles sont les relations entre mobilité et morphologie des spermatozoïdes ? La deuxième question relève des relations entre morphologie d'une part, fusion ovocytaire et fécondation d'autre part. La troisième question qui traite de l'influence de la morphologie sur le développement de l'embryon est abordée dans un autre article.

\section{a) Relation mobilité et morphologie des spermatozoïdes}

On sait que le nombre de spermatozoïdes retrouvé dans la trompe en période ovulatoire est nettement réduit par rapport au nombre observé dans l'éjaculat [47]. Dans une étude réalisée chez 5 femmes après un 
délai post coital moyen de 18 heures (10 à $35 \mathrm{~h}$ ), Ahlgren [1] retrouve un pourcentage de spermatozoïdes morphologiquement normaux plus important dans la trompe (moy $=92 \%$; extr $=89-97 \%)$ que dans le sperme $(84,5 \% ; 83-86 \%)$ recueilli 1 semaine après (voir 3 , pour une revue complète). Une filtration des spermatozoïdes s'opère au niveau du col de l'utérus, barrière physiologique dont le mucus cervical sélectionne les gamètes à morphologie normale, comme cela a été démontré par de nombreuses études (voir 27) in vivo (test post coïtal) ou in vitro (test croisé sperme-glaire). Le pourcentage de formes normales est augmenté dans le mucus cervical (par rapport à l'éjaculat), mais le pourcentage de spermatozoïdes avec des anomalies flagellaires (pièce intermédiaire ou principale) est diminué. Quant aux gamètes ayant des anomalies isolées ou multiples de la tête, ils ont une capacité de migration identique aux normaux à condition de n'avoir pas d'anomalies associées du flagelle [27].

Encore faut-il pour cela que l'analyse morphologique des spermatozoïdes soit extensive, c'est-à-dire prenne en compte les anomalies du flagelle; cette remarque pourrait expliquer pourquoi certains auteurs ont récemment rapporté que la capacité de pénétration des spermatozoides dans le mucus cervical in vitro et in vivo était négativement corrélée avec le pourcentage de têtes anormales, mais indépendantes des anomalies du flagelle [11]. Et l'exploration morphologique du flagelle reste à développer [21].

Une précision supplémentaire fut apportée par le groupe d'Overstreet \& Katz [36] qui ont montré chez des hommes féconds et inféconds, que les spermatozoïdes ayant une morphologie céphalique normale étaient plus souvent mobiles et d'une meilleure mobilité que les anormaux de la tête. Les spermatozoïdes ayant des anomalies de la tête ont plus de difficulté à traverser le mucus cervical probablement en rai- son des conséquences de leurs anomalies sur les caractéristiques de leur mobilité, avec une distance parcourue par battement flagellaire qui est plus réduite, de même que la fréquence de rotation de la tête [29, 30 ], ce qui pourrait se dire simplement de la façon suivante : un spermatozoïde morphologiquement anormal a une capacité réduite de pénétration dans le mucus cervical, même sans anomalie flagellaire. D'autres facteurs peuvent cependant intervenir; des travaux récents [9] ont montré que les différents types d'anomalies de la tête (classification OMS) étaient tous associés à une réduction significative de la vitalité, de la longévité et de l'intégrité de la membrane plasmique par rapport aux spermatozoïdes normaux.

\section{b) Relation morphologie et réaction acrosomique}

Dans le cas où les spermatozoïdes anormaux (donc principalement anomalies de la tête) traversent le mucus cervical, peuventils féconder? Il leur faut tout d'abord faire leur réaction acrosomique. La réaction acrosomique est une étape essentielle du processus de fécondation puisque seuls les spermatozoïdes ayant fait leur réaction acrosomique peuvent pénétrer la zone pellucide et fusionner avec la membrane plasmique de l'ovocyte [20]. La réaction acrosomique physiologique ne peut survenir qu'après une série de modifications des spermatozoïdes, modifications regroupées sous le terme de "capacitation". Après traitement par du fluide folliculaire humain, qui est capable d'induire la réaction acrosomique des seuls spermatozoïdes capacités, les spermatozoïdes à tête morphologiquement normale sont plus susceptibles de faire leur réaction acrosomique $(38 \%)$ que les anormaux (22\%) [20]. Une réaction acrosomique spontanée est par ailleurs retrouvée plus fréquemment pour n'importe quel type d'anomalies que pour les spermatozoïdes normaux [20, 23]. La morphologie de la tête des spermatozoïdes apparaît donc 
liée à la capacité de fécondation des spermatozoïdes.

\section{c) Relation morphologie et fécondation in vivo}

La première publication d'une relation entre morphologie des spermatozoïdes et fécondité in vivo est celle de Boskofte et al [7] qui rapporte chez 785 couples une relation significative entre l'augmentation du pourcentage de spermatozoïdes anormaux (têtes seules analysées) et la diminution de la chance d'avoir un enfant vivant, mais aussi avec le délai d'allongement du temps pour obtenir la première grossesse. Les différents types d'anomalies étudiés semblent par contre sans effet spécifique [8]. Une confirmation de la relation morphologie anormale et taux de grossesse est rapportée en 1988 par Jouannet et al [28] dans une étude prospective sur 394 couples inféconds. Deux notions nouvelles apparaissent, avec l'importance pronostique de certaines anomalies morphologiques, ainsi que de l'Index d'Anomalies Multiples (IAM). Eggert-Kruse et al [12] rapportent aussi une influence du pourcentage de spermatozoïdes anormaux ("critères stricts") sur le taux de grossesses spontanées à 1 an chez 89 couples.

\section{Indicateur de santé}

Il s'agit là des résultats de l'évaluation de quelques uns des facteurs exogènes que nous avons précédemment cités. La toxicité de certains agents chimiques ou physiques peut, comme nous l'avons vu, se traduire par des altérations de la morphologie des spermatozoïdes. De nombreuses études restent cependant à faire [26] et cela d'autant plus que si certains toxiques (ex : chaleur) n'ont un effet qu'associé à leur présence (avec un temps de rémanence), ce qui permet une récupération après suppression de l'exposition, d'autres (ex : agent orange) semblent avoir un effet qui perdure à leur présence. Dans les deux cas, la pathophysiologie doit différer.

\section{CONCLUSION}

La morphologie des spermatozoïdes est un paramètre de la spermatogenèse qui doit être étudié dans tout bilan d'infécondité de couple. L'analyse doit donner outre le pourcentage de formes normales, les différents types d'anomalies. Les informations apportées permettent d'évaluer l'état de santé du testicule et constituent l'un des facteurs prédictifs de la fertilité in vivo.

\section{BIBLIOGRAPHIE}

1. AhlgRen M. : Sperm transport to and survival in the human fallopian tube. Gynecol. Invest., 1975 ; $6: 206-214$.

2. Baccetti B, Burini AG, Collodel G, Magnano aR, Piombi P, Reneri T, SEnsini C. : Morphogenesis of the dacapitated and decaudated sperm defect in two brothers. Gamete Res. $1989 ; 23: 181-188$.

3. BarRatT CLR, Cook ID. : Sperm transport in the human female reproductive tract - a dynamic interaction. Int. J. Androl., 1991 ; 14 : 394-411.

4. Bianchi PG, Manicardi GC, URner F, Campana A, SAKKAS D. : Chromatin packaging and morphology in ejaculated human spermatozoa : evidence of hidden anomalies in normal spermatozoa. Molec. Hum. Reprod., 1996 ; 2 : 139-144.

5. BONDE JP. : Semen quality and sex hormones among mild steel and stainless steel welders : a cross sectional study. Br. J. Ind. Med., $1990 ; 47$ : 508-514.

6. BONDE JPE. : The risk of male subfecundity attributable to welding of metals. Int. J. Androl., 1993 ; 16 : suppl $1: 1-29$.

7. BOSTOFTE E, SERUP J, REBBE H. : Relation between morphologically abnormal spermatozoa and pregnancies obtained during a twenty-year follow-up period. Int. J. Androl., 1982 ; 5 : 379-386.

8. Bostofte E, Serup J, Rebbe H. : The clinical value of morphological rating of human spermatozoa. Int. J. Fertil., $1985 ; 30: 31-37$.

9. Carrell DT, Zobell RL, Middletown RG, URry LR. : A functionnal analysis and the potential clinical significance of 7 categories of sperm morphology. J. Urol., 1994 ; $151: 376-380$.

10. Destefano F, Annest JL, Kresnow MJ, Schrader SM, KaTZ DF. : Semen characteristics of Vietnam veterans. Reprod. Tox., $1989 ; 3: 165-173$. 
11. Eggert-Kruse W, Reinman-Andersen J, Rohr G, Pohl S, Tilgen W, Runnebaum B. : Clinical relevance of sperm morphology assessment using strict criteria and relationship with sperm-mucus interaction in vivo and in vitro. Fertil. Steril., 1995 ; $63: 612-624$.

12. Eggert-Kruse W, Schwarz H, Rohr G, Demisakca T, Tilgen W, Runnebaum B. : Sperm morphology assessment using strict criteria and male fertility under in-vivo conditions of conception. Hum. reprod., $1996 ; 11: 139-146$.

13. ESCALIER D. : Human spermatozoa with large heads and multiple flagella : quantitative ultrastructural study of 6 cases. Biol. Cell, 1983, 48 : 65-74.

14. ESCALIER D. : Failure of differentiation of the nuclear perinuclear skeletal complex in the roundheaded human spermatozoa. Int. J. Dev. Biol., $1990,34: 287-297$.

15. Escallier D, David G. : Pathology of the cytoskeleton of the human pserm flagellum: axonemal and peri-axonemal anomalies. Biol. Cell, 1984, 50 : 37 52.

16. Figa-Talamanca I, Delli'Orco V, Pupi A et al. : Fertility and semen quality of workers exposed to high temperature in the ceramic industry. Reprod. Toxicol., $1992 ; 6: 517-523$.

17. Flörke-Gerloff S, Topfer-Petersen E, MưllerESTERL W ET AL. : Biochemical and genetic investigation of roundheaded spermatozoa in infertile men including two brothers and their father. Andrologia, $1984 ; 16: 187-202$

18. Fredericsson B. : On the development of different morphologic abnormalities of human spermatozoa. Andrologia, $1978 ; 10: 43-48$.

19. FrIT/ G, GzErzel AE. : Abnormal sperm morphology and function in the fathers of hypospadias. $\mathrm{J}$. Reprod. Fertil., 1996 ; 106 : 63-66.

20. Fukuda M, Morales P, Overstreet JW. : Acrosomal function of human spermatozoa with normal and abnormal head morphology. Gam. Res., 1989 ; $24: 59-65$

21. Gomez E, Buckingham DW, Brindele J, Lanzafame F, IRvine DS. AITKEN JR. : Development of an image analysis system to monitor the retention of residual cytoplasm by human spermatozoa : correlation with biochemical markers of the cytoplasmic space, oxydative stress, and sperm function. J. Androl., 1996; 17: 276-287.

22. Hanjeisman DJ, Convay aJ, Boylan LM, Turtle JR. : Testicular function in potential sperm donors : normal range and the effects of smoking and varicocele. Int. J. Androl., $1984 ; 7$ : 369-382.
23. Heywinkel E, Freundl G, Hofmann N. : Acrosome reaction of spermatozoa with different morphology. Andrologia, $1993 ; 25: 137-139$.

24. HRUdKA F, Sinch A. : Sperm nucleomalacia in men with inflammatory bowel disease. Arch. Androl., $1984 ; 13: 37-57$.

25. Hudson E, Dore G, Sowter C, Toovey S, Levi AJ : Sperm size in patients with inflammatory bowel disease on sulfasalazine therapy. Fertil. Steril., $1982 ; 38: 77-84$.

26. JAHN AI, GünZEL PK. : The value of spermatology in male reproductive toxicology : do spermatologic examinations in fertility studies provide new and addition of information relevant for safety assess ment? Reprod. Toxicol., 1997, 11 : 171-178.

27. Jeulin C, Soumah A, Jounnnet P. : Morphological factors influencing the penetration of human sperm into cervical mucus in vitro. Int. J. Androl., $1985 ; 8: 215-223$.

28. Jounannet P, Ducot B, Feneux D, Spira A. : Male factors and likelihood of pregnancy in infertile couples. I. Study of sperm characteristics. Int. J. Androl., $1988 ; 11$ : 379-394.

29. Katz DF, Diel L, OverstreEt JW. : Differences in the movement of morphologically normal and abnormal human seminal spermatozoa. Biol. Reprod., $1982 ; 26$ : 566-570.

30. Katz DF, Morales I, Samules SJ, Overstreet JW : Mechanisms of filtration of morphologically abnormal human sperm by cervical mucus. Fertil. Steril., $1990 ; 54$ : 513-516.

31. Lancranjan I, Popescu HI, Kuepsch I. : Changes of the gonadic function in chronic carbon disulphide poisoning. Med. Lav., 1969 ; 60 : 566-571.

32. MACLEOD J. : Seminal cytology in the presence of varicocele. Fertil. Steril., $1965 ; 16$ : 735-739.

33. Martin RH, Rademaker A. : The relationship between sperm chromosomal abnormalities and sperm morphology in humans. Mut. Res., 1988 ; 207 : 159-164.

34. MeYer CR. : Semen quality in workers exposed to carbon disulphide compared to a control group from the same plant. J. Occup. Med., $1981 ; 23$ : $435-439$

35. Mieusset R, Bujan L, Mansat A, Pontonnier F, GRANDJEAN H. : Effects of artificial cryptorchidism on sperm morphology. Fertil. Steril., $1987 ; 47$ : 150-155.

36. Morales P, Katz DF, Overstreet JW, Samuels SJ, Chang RJ. : The relationship between the morphology of spermatozoa in human semen. J. Androl., 1988 ; 9 : 241-247. 
37. Overstreet JW, Price MJ, Blazak WF, Lewis EL, KATZ DF. : Simultaneous assessment of human sperm motility and morphology by videomicrography. J. Urol., $1981 ; 126 ; 357-360$.

38. Procope BJ. : Effect of repeated increase of body temperature on human sperm quality. Int. J. Fertil., $1965 ; 10: 333-339$.

39. Ratcliffe JM, Schrader SM, SteEnland K, ClapP DE, TuRner T, Hornung RW. : Semen quality in papaya workers with long term exposure to ethylene dibromide. Br. J. Ind. Med., 1987 ; 44 : 317-326.

40. Riley SA, Lecarpentier J, Mani V, Goodman MJ, MANDAL BK, TURnBerg LA. : Sulphasalazine induced seminal abnormalities in ulcerative colitis : results of mesalazine substitution. Gut., $1987 ; 28$ : 1008-1012.

41. Rock J, Robinson D. : Effect of induced scrotal hyperthermia on testicular function in man. Am. J. Obstet. Gynec., $1965 ; 93: 793-801$.

42. Ross A, Christie S, Edmond P. : Ultrastructural tail defects in the spermatozoa from two men attending a suberfertility clinic. J. Reprod. Fertil. $1973 ; 32: 243-251$.

43. Schrader SM, Ratcliffe JM, Turner TN, HorNUNG RW. : The use of new field methods of semen analysis in the study of occupational hazards to reproduction : the example of ethylene dibromide. J. Occup. Med., 1987 ; 29 : 963-966.

44. Schrader SM, TURner TW, Simon SD. : Longitudinal study of semen quality of unexposed workers : sperm head morphometry. J. Androl., 1990 ; 11 : 32-39.

45. Schwartz D, Ducot B, Auroux M, Collin M. : Within-subject variability of the percentage of morphologically abnormal spermatozoa among fertile men : biological and measurement components. Hum. Reprod., 1986 ; 1 : 369-371.

46. Toтн A. : Reversible toxic effect of salicylazosulfapyridine on semen quality. Fertil. Steril., $1979 ; 31$ : $538-540$.

47. Williams M, Hill CJ, Scudzmore I, Dunphy B, CoOKe ID, BarratT CLR. : Sperm numbers and distribution within the human fallopian tube around ovulation. Hum. Reprod., 1993 ; 8 : 20192026.

48. Wyrobek AJ, Watchmaker G, Gordon L, Wong K, MoORE D, WhorTon D. : Sperm shape abnormalities in carbaryl-exposed employeers. Environ. Health Perspec., 1981 ; 40 : 255-265.

49. ZAMBONI L. : Sperm stracture and its relevance to infertility. Arch. Pathol. Lab. Med., 1992, 116 : 325-344.

\section{ABSTRACT \\ Sperm morphology \\ R. Mieusset}

Spermatozoa morphology is one of the qualitative characteristics of spermatogenesis. However, because of both the variations in the definition of normal morphology and the existence of different kinds of sperm abnormalities as well as the use of various techniques of morphology assessment, such a parameter is poorly used in usual laboratory work. Morphological sperm anomalies can be from testicular or post-testicular origines, while the latter is still unproved. The causes of such anomalies are either from genetic origines, but in these cases any spermatozoa demonstrate this anomaly, or due to an endogenous factor with varicocele the most usually quoted but unproved pathology, But exogenous factors, either chemical such as drugs and pesticides or physical such as heat, are also responsible for morphological sperm anomalies. Analysis of sperm morphology is indicative of both the testicular health status (in cases of occupational exposure to chemical or physical toxics) and the fertility potential since morphology is correlated to sperm motilily and involved in fertilization through the acrosome reaction.

Key Words : spermatozoa, morphology, fecundity, toxicology. 